\title{
The Prospective Role of Cognitive Appraisals and Social Support in Predicting Children's Posttraumatic Stress
}

\author{
Caitlin Hitcheock $^{1,2}$ - Alicia A. Ellis ${ }^{2}$ Paul Williamson ${ }^{2} \cdot$ Reginald D. V. Nixon $^{2}$
}

Published online: 14 May 2015

(C) Springer Science+Business Media New York 2015

\begin{abstract}
Although both social support and cognitive appraisals are strong predictors of children's posttraumatic adjustment, understanding of the interplay between these factors is limited. We assessed whether cognitive appraisals mediated the relationship between social support and symptom development, as predicted by cognitive models of posttraumatic stress disorder (PTSD). Ninety seven children (Mean age $=$ 12.08 years) were assessed at one month and six months following a single incident trauma. We administered self-report measures of cognitive appraisals, social support, and a diagnostic interview for PTSD. Results indicated that cognitive appraisals at one month post-trauma mediated the relationship between social support at one month post-trauma, and PTSD severity at follow-up. Differences in this relationship were observed between child-reported social support and parentrated ability to provide support. Firm evidence was provided for the application of cognitive models of PTSD to children.
\end{abstract}

Electronic supplementary material The online version of this article (doi:10.1007/s10802-015-0034-7) contains supplementary material, which is available to authorized users.

Caitlin Hitchcock

Caitlin.hitchcock@mrc-cbu.cam.ac.uk

Reginald D. V. Nixon

Reg.nixon@flinders.edu.au

Alicia A. Ellis

a.ellis@internode.on.net

Paul Williamson

Paul.williamson@flinders.edu.au

1 MRC Cognition and Brain Sciences Unit, 15 Chaucer Road, Cambridge CB2 7EF, UK

2 School of Psychology, Flinders University, GPO Box 2100, Adelaide, SA 5001, Australia
Keywords PTSD $\cdot$ Children $\cdot$ Cognitive appraisals $\cdot$ Social support

Childhood trauma exposure can have a debilitating impact on emotional health. Previous research demonstrates that the development of acute and posttraumatic stress following childhood trauma exposure is influenced by trauma type, along with characteristics of the child at the time of the trauma (for review see Alisic et al. 2011). However, post-event factors, particularly acute reactions, also have a significant impact on prognosis (Alisic et al. 2011; Trickey et al. 2012). A recent meta-analysis indicated that two of the largest post-event predictors of outcome were the appraisals a child makes of the event (e.g., blaming others), and the support they receive from others (Trickey et al. 2012).

Cognitive models of posttraumatic stress disorder (PTSD) propose that appraisals of the traumatic event are critical influences in posttraumatic responses (Ehlers and Clark 2000). In particular, negative appraisals about the traumatic event, and about one's posttraumatic symptoms and the reaction of others following the trauma are paramount in explaining the development and maintenance of PTSD. This may be due to negative appraisals fostering avoidance and hindering reprocessing of the trauma, and maintaining a negative sense of self. Evidence in adults for the role of negative appraisals in predicting PTSD is well developed (Dalgleish et al. 2005). An increasing number of studies in children demonstrate the detrimental impact of negative appraisals on emotional health following trauma. Meiser-Stedman et al. (2007) demonstrated a strong relationship between maladaptive appraisals and acute posttraumatic stress in 10-16 year old motor vehicle accident/assault victims. Similarly, Salmon et al. (2007) reported that negative appraisals of personal vulnerability and future harm accounted for unique variance in posttraumatic 
stress in the acute phase following trauma. Further, Stallard and Smith (2007) reported that negative appraisals of symptoms, a sense of injustice, and belief of permanent change for the worse were also cross-sectionally associated with symptoms at eight months post-trauma.

Stronger support is provided by prospective studies. An initial study by Ehlers et al. (2003) indicated that negative interpretation of intrusions measured at three months predicted PTSD symptoms at six months post-trauma. More comprehensively, Bryant and colleagues (2007) examined the predictive power of appraisals immediately following trauma on longer-term outcomes for children admitted to hospital following a traumatic injury. Their results demonstrated that negative appraisals of vulnerability in the first month following trauma, indexed using the Child Posttraumatic Cognitions Inventory (CPTCI; Meiser-Stedman et al. 2009 ${ }^{1}$ ), uniquely predicted posttraumatic stress at six months post-trauma. This effect was found to be independent from, and of greater predictive power than, child age, acute posttraumatic stress symptoms, injury severity score, and parental posttraumatic stress. This finding was replicated and extended by MeiserStedman et al. (2009). The authors measured negative appraisals at 2-4 weeks, and then again six months posttrauma using the CPTCI. Results demonstrated that negative appraisals in the acute phase, namely the Permanent and Disturbing Change subscale of the CPTCI, mediated the relationship between acute stress symptoms and PTSD symptoms at six month follow-up, even after controlling for symptoms at the acute stage. This finding was consistent with Ehlers and Clark's (2000) model, which proposed that negative interpretations of one's reaction to the trauma are central in the development of posttraumatic stress symptoms. In sum, negative appraisals in the weeks following trauma are a robust predictor of later adjustment.

Although negative appraisals are consistently implicated in emotional wellbeing following trauma, there is less investigation of the possible protective function of adaptive appraisals. In considering the risk factors discussed above, it is reasonable to hypothesise that adaptive appraisals may positively influence prognosis. Adaptive appraisals are not simply the absence of negative appraisals, but particular thoughts and beliefs that, by their presence, may protect against psychopathology. Adaptive appraisals after trauma may include the belief that everything will be alright, acceptance (rather than avoidance) of the traumatic event, or the belief that posttraumatic stress symptoms are acceptable following a trauma. Given that appraisals are integral in the development of PTSD, and negative appraisals are associated with poorer outcome, it is important to determine whether adaptive appraisals impact

\footnotetext{
${ }^{1}$ At the time of Bryant et al.'s (2007) paper, the CPTCI was an unpublished measure. A validation of the CPTCI was published in 2009.
}

prognosis. For example, as negative beliefs regarding selfefficacy (i.e., personal weakness) are related to heightened symptoms (Benight and Bandura 2004), adaptive appraisals of self-efficacy (e.g., I will get over this) may protect against symptoms. There is currently no direct research on the impact of adaptive appraisals following trauma. In anxiety research more broadly, adaptive self-statements (e.g., I am brave) are related to better emotional functioning (Ronan et al. 1994), as are ratings of one's self-efficacy (Rudy et al. 2012) and perceived self-competence (Uhrlass et al. 2009). When considering the role of appraisals in PTSD development, examination of trauma-specific adaptive appraisals is clearly warranted as directly encouraging adaptive appraisals in the first months following trauma may significantly impact longer term prognosis.

Given the important role of appraisals in predicting PTSD, elaboration of appraisal development also has critical clinical implications. One potential influence on posttraumatic appraisals is social support (Ehlers and Clark 2000). There is minimal direct research that simultaneously examines social support and posttraumatic appraisals (whether negative or adaptive), however it is likely that social support immediately post-trauma may impact later symptoms through such appraisals. Discussion with others, or hearing adults' appraisals of the event, may provide a means of reappraising the event and correcting any misinterpretations. Indeed, it has been previously demonstrated that parents are important in helping children to appraise an event in a helpful way. If the parent does not appraise the event in a helpful way themselves, or is unavailable to provide this support, there may be an increased risk of the child developing posttraumatic stress (MeiserStedman 2002). Similarly, parent response is crucial in minimising posttraumatic stress following children's disclosure of sexual abuse, arguably through influencing appraisals (Spaccarelli 1994). Children may receive social support from a variety of sources. It is therefore worthwhile to assess social support from a range of people in addition to assessing support from parents specifically. Given the importance placed on social support in the aftermath of trauma (National Institute for Health and Care Excellence 2005) and the proposed conceptual link between social support and appraisals (and subsequently symptoms), it is somewhat surprising that there is a dearth of research assessing these variables simultaneously.

We prospectively assessed predictors of childhood PTSD, with a particular focus on the role of negative and adaptive appraisals of the trauma, and whether these appraisals mediated the relationship between social support in the month following trauma and later symptomatology. We have previously published a cross-sectional examination of whether negative appraisals mediated the impact of social support on acute stress symptoms in the first month following trauma (Ellis et al. 2009). In that paper, Baron and Kenny's (1986) prerequisites for mediation were not met; thus the mediation analysis 
was not completed. In cases when the independent variable does not uniquely predict the outcome variable (as occurred in Ellis et al. 2009), an indirect effect may be erroneously rejected under the prerequisites outlined by Baron and Kenny (Preacher and Hayes 2004; Hayes 2013). Explicit assessment of an indirect relationship using recent analytical techniques (e.g., Process; Preacher and Hayes 2004) reduces such Type II error, and will more accurately assess mediation than a series of separate significance tests (Hayes 2009; Hayes and Scharkow 2013; Preacher and Hayes 2004). Advancement in statistical methods therefore indicate that re-evaluation of the proposed mediation is warranted.

Our key aim was to extend our current knowledge of this topic by examining whether appraisals mediated the impact of acute social support on posttraumatic stress six months later. We also provide a preliminary examination of the relationship between adaptive appraisals and prognosis. In doing so, we answer two key questions. First, do cognitive appraisals mediate the effect of social support on both short and longer term functioning? Second, do adaptive appraisals uniquely influence post-traumatic adjustment, separate from the effect of negative appraisals? We hypothesised that the effect of social support on PTSD symptoms would be mediated by cognitive appraisals. We also hypothesised that both acute negative and adaptive appraisals would uniquely influence PTSD symptoms at six months post-trauma, such that negative appraisals would predict elevated symptoms and adaptive appraisals would predict fewer symptoms.

\section{Method}

\section{Participants}

Young people aged 7-17 years were recruited from two metropolitan hospitals, where they were treated following a single incident trauma (e.g., road traffic accident, see Table 1 for participant characteristics). Exclusion criteria included nonCriterion A trauma (e.g., minor fall), lack of English fluency, traumatic brain injury, ongoing treatment that was potentially traumatic in nature (e.g., burns treatment), or ongoing trauma (e.g., domestic violence). A total of 325 families were identified, of which 175 met exclusion criteria, predominately due to young age. Of the 148 children remaining, 51 declined participation. The final sample therefore consisted of 97 children.

\section{Materials}

Trauma Interview Parents reported demographic information, child's prior trauma history, previous psychological or pharmacological treatment for emotional problems, and family history of psychological problems. Length of hospitalisation was also recorded, along with parental rating of injury severity on a 4-point scale where $0=$ no injury, $1=$ minor injuries (e.g., bruises/abrasions), 2=moderate injuries (e.g., broken bones) and $3=$ severe injuries (e.g., multiple fractures, internal injuries). Parents also identified whether their
Table 1 Sample characteristics $(N=97)$

\begin{tabular}{|c|c|c|c|c|}
\hline Variable & Mean & $S D$ & Min & $\operatorname{Max}$ \\
\hline Child age in years & 12.08 & 2.80 & 7 & 17 \\
\hline Gender & $63 \% \mathrm{M}(n=61)$ & & & \\
\hline Length of hospitalisation (days) & 6.69 & 14.09 & 0 & 120 \\
\hline Injury severity & 1.82 & 0.92 & 0 & 3 \\
\hline Type of trauma & $47 \%(n=46)$ & & & \\
\hline Road traffic accident & $12 \%(n=12)$ & & & \\
\hline Significant fall & $13 \%(n=13)$ & & & \\
\hline Serious accidental injury & $27 \%(n=26)$ & & & \\
\hline Other(assault/medical emergency/house fire) & & & & \\
\hline Prior trauma exposure ${ }^{a}$ & $42 \%(n=41)$ & & & \\
\hline Prior psychological treatment ${ }^{a}$ & $24 \%(n=23)$ & & & \\
\hline Prior pharmacological treatment & $1 \%(n=1)$ & & & \\
\hline Child psychological treatment since trauma & $6.2 \%(n=6)$ & & & \\
\hline Family history of psychological problems ${ }^{\text {a }}$ & $18 \%(n=17)$ & & & \\
\hline Child Posttraumatic Stress Scale at one month & 11.16 & 9.87 & 0 & 42 \\
\hline CAPS symptom severity at follow-up & 13.22 & 13.15 & 0 & 59 \\
\hline Child Depression Inventory at one month & 7.96 & 6.64 & 0 & 30 \\
\hline Child Depression Inventory at follow-up & 5.28 & 6.53 & 0 & 41 \\
\hline
\end{tabular}

a Variable coded dichotomously; M Male; CAPS Clinician Administered PTSD Scale (Nader et al. 1994) 
child had received medication or psychological treatment following the trauma.

Symptom Measures The Clinician Administered PTSD Scale for Children (CAPS; Nader et al. 1994) is the goldstandard diagnostic tool for PTSD in children. In addition to diagnostic status, the scale yields a score for symptom severity, which considers the frequency and intensity of symptoms. Inter-rater reliability of $15 \%$ of assessments was completed by an independent rater, resulting in $100 \%$ agreement on diagnostic status. Children also completed the Child Posttraumatic Stress Scale (CPSS; Foa et al. 2001), and the Child Depression Inventory (CDI; Kovacs 1992). Internal consistency was high for both measures $(\alpha=0.88$ and $\alpha=$ 0.92 , respectively).

Negative Appraisals The Child Posttraumatic Cognitions Inventory (CPTCI: Meiser-Stedman et al. 2009) is a 25- item self-report measure of children's negative appraisals about the trauma and its consequences. Items are separated into two subscales; Disturbing and Permanent Change, and Feeble Person in a Scary World. The total score for the combined subscales was used in all analyses. Internal consistency was high, $\alpha=.95$.

Adaptive Appraisals In the absence of an established measure of adaptive cognitive appraisals that children may have following a traumatic event, a specific scale was developed. The Adaptive Appraisals Questionnaire (AAQ) consists of 21 self-report items, with items derived from theory (Ehlers and Clark 2000), therapy guidelines (Perrin et al. 2004) and previous research (Ehlers et al. 2003). Questions were designed to assess a child's appraisals of wellness (Hasan and Power 2004); positive change following the event (Linley and Joseph 2004); current threat (Ehlers and Clark 2000); omen formation (Meiser-Stedman et al. 2009); appraisals about the trauma and symptoms following the trauma, other people's responses to the trauma (Ehlers and Clark 2000); self-blame (Cohen et al. 2004) and appraisals of the usefulness of talking about the event (Meiser-Stedman 2002). Initially, 103 items were generated, following which items similar to the CPTCI were deleted. Subsequently, expert opinion was sought from four clinical psychologists with experience working with children, and 2 developmental psychologists. Confusing or repetitive items were deleted and the remaining questions were trialled with eight children and adolescents. The final 21 items are scored on a 4 - point scale $(1=$ don't agree at all, $4=$ agree $a$ lot $)$. Internal consistency was satisfactory, $a=0.80$. A moderate correlation with the CPTCI indicated that the AAQ was not simply an inverse measure of negative appraisals, although there was overlap in variance between the measures, $r=-0.57, n=97, p<0.001$. The final measure is available in the online supplementary materials.
Social Support Parents' perceptions of how well they supported their child were measured using two subscales of the Parent-Child Relationship Inventory (PCRI: Gerard 1994). The PCRI-Support and PCRI-Communicate subscales consist of nine items each, which were summed to provide an overall measure of parent availability to provide support to their child. A higher score denotes a higher level of parent availability to provide support. In the present sample internal consistency for the combined subscales was satisfactory, $\alpha=0.75$.

The child's perception of social support was measured using the Multidimensional Scale of Perceived Social Support (MSPSS; Zimet et al. 1988). The scale consists of 12 items reflecting perceived support from three areas: family, a special person, and friends. To increase its utility for younger children, we added clarification in parentheses to some items. For example: 'I have friends with whom I can share my joys and sorrows (happy and sad things)'. Scores were summed and a higher score reflected greater social support. The MSPSS has demonstrated reliability and validity (CantyMitchell and Zimet 2000). In the present study internal consistency was high, $\alpha=0.90$.

\section{Procedure}

This study was approved by hospital ethics committees. Children were considered for eligibility, following which a researcher either approached the family while they were at the hospital, or telephoned following discharge. Informed consent was obtained from all individual participants included in the study. Parents and children then completed assessments either in a face-to-face interview or by telephone and post (for those already discharged). Interviews were conducted by trained clinical staff under the supervision of R.D.V. Nixon. Participants received a cinema ticket or $\$ 10$ shopping voucher. In the acute phase (within one month of trauma; T1), the trauma interview and self-report measures were completed. At six months post-trauma (T2), the CAPS and self-report measures were completed.

\section{Results}

\section{Sample Characteristics}

Sample characteristics are presented in Table 1, as are symptom scores at T1 and T2. Attrition saw that at follow-up, 90 children completed diagnostic interviews and 84 completed the self-report measures. Child-reported posttraumatic stress symptoms at T1 (indexed on the CPSS) was related to both child-reported symptoms at T2, $r=0.41, n=78, p<.001$, and clinician-rated symptoms at $\mathrm{T} 2$ (indexed by total severity score on the CAPS), $r=0.27, n=87, p=.01$. 


\section{Hypothesis Testing}

Indirect effects were assessed using the Process statistical package, creating 10,000 bootstrapped samples for each analysis. Process allowed us to determine the direct effect of the independent variable (IV) on the dependent variable (DV), and an indirect effect of the IV on the DV through one or more proposed mediators. The indirect effect represents the multiplication of the unstandardised effect of the IV on the M, with the effect of the $\mathrm{M}$ on the DV. We determined the significance of an indirect effect using $95 \%$ bias-corrected confidence intervals. The unstandardised regression coefficient for the indirect effect (i.e., the $b$ coefficient) is considered significant if the associated confidence interval does not span zero. Standard error is presented for each coefficient $(S E b)$. Model statistics for significant indirect effects are presented in Table 2.

First we determined whether cognitive appraisals mediated the relationship between social support and posttraumatic stress (measured using the CPSS) at one month post-trauma. We began with perceived social support and determined whether scores on the CPTCI mediated the relationship between MSPSS at T1 and CPSS at T1 (see Model 1 in Table 2). A significant indirect effect supported our hypothesis. That is, negative appraisals mediated the relationship between perceived social support (child report) and posttraumatic stress in the acute phase following trauma. ${ }^{2}$ When this model was repeated with parent-reported support as the predicting variable, no indirect effect was evident, $b=-0.17, S E b=0.10$, $[-0.37,0.01]$.

The above analyses were repeated with AAQ as the mediator to examine whether adaptive appraisals also mediated the relationship (Model 2). Again, a significant indirect effect was observed. Consistent with our hypothesis, adaptive appraisals mediated the relationship between perceived social support and posttraumatic stress. This relationship was not observed for parent-reported support, $b=-0.13, S E b=0.13,[-0.43$, $0.09]$.

To determine whether the effect of adaptive appraisals was independent from negative appraisals, we next completed a parallel mediation model, with AAQ and CPTCI entered as simultaneous mediators (Model 3). The model demonstrated that CPTCI remained a significant mediator of the impact of perceived social support on symptoms one month post-trauma, however, the AAQ did not. Neither of the appraisal measures mediated the effect of parent-reported support, AAQ $b=$

${ }^{2}$ This relationship was not analysed initially by Ellis et al. (2009), as Baron and Kenny's (1986) perquisites for mediation were not met. The observed mediation in the current analysis reflects the implications of new conceptual understanding and practical applications of mediation testing (Hayes 2013).

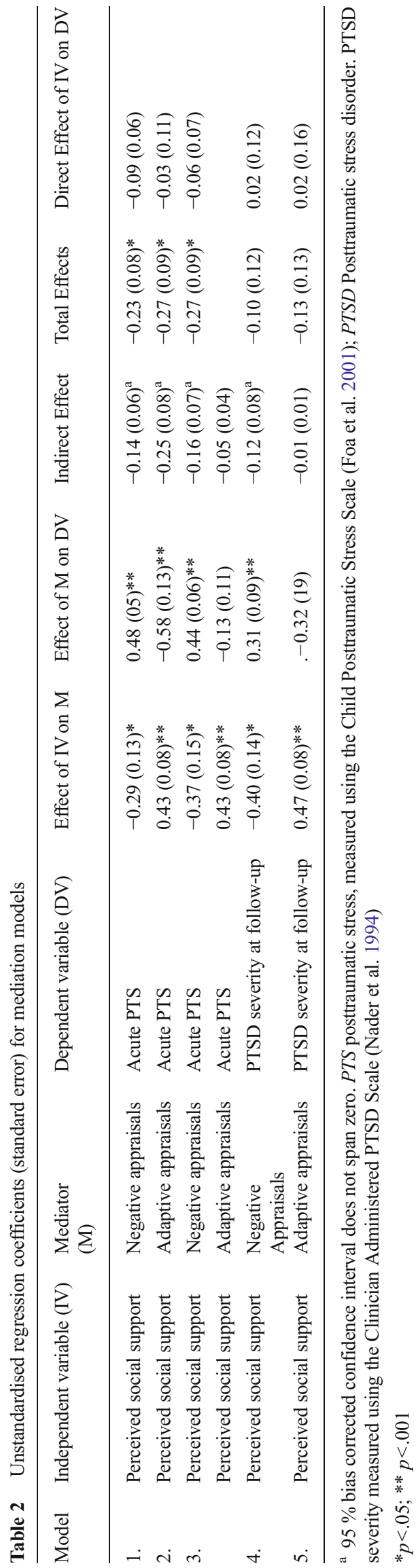


$-0.04, S E b=0.05,[-0.19,0.02]$; CPTCI $b=-0.16, S E b=0.10$, $[-0.39,0.02]$.

Next we examined the prospective data to determine whether appraisals at $\mathrm{T} 1$ mediated the impact of perceived social support at T1 on PTSD symptoms six months later. CAPS severity score (measured at T2) was entered as the dependent variable, MSPSS at T1 was entered as the independent variable, and the appraisals measure was entered as the mediator. Negative appraisals did significantly mediate the effect of perceived social support on later PTSD (Model 4). In a separate model, no evidence was found for AAQ as a mediator (Model 5). That is, negative appraisals mediated the impact of perceived social support on longer term functioning, but adaptive appraisals did not.

The above models were repeated with parent-reported support as the predicting variable. Replicating the relationship observed at T1, no mediation was observed through AAQ, $b=-0.07, S E b=0.09,[-0.37,0.04]$, or CPCTI, $b=-0.10$, $S E b=0.08,[-0.36,0.01]$.

We also hypothesised that appraisals in the acute phase would independently predict later posttraumatic stress. This hypothesis was assessed by examining the unique effect of appraisals (i.e., the mediator) on symptom severity (i.e., the dependent variable) in the mediation analyses completed above. Replicating previous research, negative appraisals at T1 significantly predicted later PTSD severity (see Model 4). However, adaptive appraisals did not account for unique variance in later symptom severity (see Model 5). It is possible that adaptive appraisals predicted symptoms at $\mathrm{T} 1$ but not at $\mathrm{T} 2$ due to use of self-reported symptoms (i.e., CPSS) as the outcome at $\mathrm{T} 1$ and use of clinician-rated symptoms (i.e., CAPS severity score) as the outcome at T2. We therefore repeated analyses with CPSS at T2 as the outcome. Consistent with results for clinician-rated symptoms, negative appraisals significantly predicted self-report symptoms, $b=$ $0.15, S E b=0.05,[0.04,0.26]$, but adaptive appraisals did not, $b=-0.06, S E b=0.12,[-0.30,0.18]$. There was also no direct effect of perceived social support on self-report symptoms, $b=-0.04, S E b=0.07,[0.04,0.26]$.

\section{Discussion}

This study demonstrated that perceived social support and negative appraisals in the acute phase following trauma will have a significant impact on longer term functioning following a childhood trauma that has resulted in hospital attendance. Specifically, negative appraisals mediated the relationship between perceived social support and posttraumatic stress at both one month and six months post-trauma. These results extend understanding of how support from important people in the child's life can influence psychological wellbeing post-trauma, and also replicate previous findings of the importance of negative appraisals in the development of PTSD. Importantly, given that cognitive models of PTSD were developed to explain adult psychopathology, we have provided additional support for the application of cognitive models of PTSD to children (e.g., Brewin et al. 1996; Ehlers and Clark 2000).

Cognitive models of PTSD define negative appraisals as integral in the aetiology of PTSD. Our results supported this proposition, replicating previous support for the role of acute negative appraisals in symptom development (Bryant et al. 2007; Meiser-Stedman et al. 2009). We have also expanded understanding of cognitive models by exploring how social support contributes to the development of appraisals. Ehlers and Clark (2000) asserted that interpretations regarding the support of others will impact appraisal development. For example, when people avoid talking about the event so as not to upset the individual with PTSD, this may lead the individual to perceive that others think the trauma is their own fault. Our findings were consistent with interpretations of support impacting appraisal development. Our measure of perceived social support indexed whether the child felt they could discuss the trauma with significant others, and consistent with the cognitive model's proposition, this was negatively related to negative appraisals. In turn, this related to fewer PTSD symptoms at follow-up, as predicted by the model. Our findings therefore provide firm support for the cognitive model of PTSD in children.

Our findings also extend understanding of how social support influences symptom development. Interestingly, social support had a moderate impact on symptoms in the acute phase post-trauma, but only indirectly impacted longer term adjustment through influencing negative appraisals. This finding emphasises the complex relationship between social support and child wellbeing, and highlights the need to explore mechanisms through which social support will impact posttraumatic adjustment. Our results also suggest that the child's perception of social support will play a larger role in symptom development than the parent's report of support for their child. This raises two key issues; that it is important to consider the child's perception of support, rather than relying on parent report, and that the wider system of support around the child, including friends and significant persons, may be important determinants of a child's wellbeing post-trauma. The MSPSS asks whether the child can discuss their difficulties with their friends, and important people (e.g., siblings, grandparents) in their life, rather than focussing exclusively on the parent-child relationship as indexed by the PCRI. The larger effect of the MSPSS and mediation via negative appraisals may suggest that wider social support, and/or the child's perception, rather than parental belief in their ability to provide support, are particularly important in symptom development. This point was emphasised in recent meta-analysis (Alisic et al. 2011). Our results support the consideration of the wider support 
system around the child. In particular, early clarification of children's perception of social support may assist such that if there was little perceived support, early intervention in a systems approach to engage available supports may be beneficial. Assessment with objective measures of social support (e.g., coding of observed interactions) may improve understanding of these relationships.

Regarding appraisals more specifically, we examined whether early adaptive appraisals post-trauma impacted wellbeing both cross-sectionally and prospectively. Our findings demonstrated that adaptive appraisals were inversely related to acute symptoms, but did not predict longer term functioning. However, the effect of adaptive appraisals on acute symptoms was no longer present once variance explained by negative appraisals was removed. This may suggest that adaptive appraisals are non-distinct from negative appraisals in a clinical sample. The ability to draw such a conclusion from the current data is limited, as the AAQ we developed in the absence of a published measure of adaptive appraisals may have contributed to overlap between adaptive and negative appraisals. Alternatively, it is possible that the two are indeed separate constructs, but in the case of posttraumatic adjustment, negative rather than adaptive appraisals are more influential. Consistent with the weakest link hypothesis, maladaptive cognitions may outweigh the protective benefit of adaptive appraisals (Abela and Sarin 2002). In turn, adaptive appraisals may predict posttraumatic growth (which we did not measure), while negative appraisals predict posttraumatic stress. Theories of PTSD aetiology have focussed on the role of negative appraisals, and as a result, exploration of adaptive appraisals in symptom development is currently limited. Given the clinical emphasis on encouraging adaptive appraisals in treatment of psychopathology (e.g., through cognitive restructuring) and the importance of considering positive factors post-trauma (Alisic et al. 2011) further exploration of adaptive appraisals is warranted. In particular, future research to develop and validate measures of adaptive appraisals may improve understanding of the interrelationship between adaptive and negative cognitions. Further exploration of the relative impact of appraisals, both adaptive and negative, in samples with more chronic trauma exposure such as childhood abuse may clarify these issues.

Finally, our results offer implications for the treatment of childhood PTSD. First, the prospective effects of perceived social support (and not parent-reported support) in the shortterm following trauma indicates that it would be beneficial to encourage supportive relationships not only between the child and parent, but also within the child's wider system. Given the hypothesised role of significant others in appraisals of the traumatic event, it may also be beneficial for therapy to involve significant people in the child's life. This does occur in some treatments of child PTSD (e.g., trauma-focussed cognitive behavioural therapy), but in particular, it may be helpful to discuss with the child's 'special person' how they may assist the child to appraise the event in a non-catastrophising manner. When completing cognitive restructuring with traumaexposed children, exploration of how beliefs are impacted by others is also likely to be beneficial. Indeed, previous examination of parental appraisal of the trauma has indicated a key role in child appraisals of the event (Morris et al. 2013). Based on our findings, we encourage the consideration of those identified by the child as providing support, in addition to the child's parents.

In conclusion, this study has filled a noticeable gap in the literature regarding how social support relates to the development of appraisals and subsequent PTSD symptoms in children. Our findings highlight that both social support and negative appraisals in the acute phase following trauma influence longer term functioning, and suggest that further exploration of adaptive appraisals may be warranted. The need for interventions enhancing adaptive appraisals has been emphasised (Bryant et al. 2007), however, we suggest that any differential impact of adaptive and negative appraisals on PTSD symptoms must first be established.

Acknowledgments This study was funded by grants from the Australian Research Council (DP 0771885) and the Australian Rotary Health Research Fund awarded to the last author.

Conflict of Interest The authors declare that they have no conflict of interest.

\section{References}

Abela, J., \& Sarin, S. (2002). Cognitive vulnerability to hopelessness depression: a chain is only as strong as its weakest link. Cognitive Therapy and Research, 26, 811-829. doi:10.1023/A:1021245618183.

Alisic, E., Jongmans, M. J., van Wesel, F., \& Kleber, R. (2011). Building child trauma theory from longitudinal studies: a meta-analysis. Clinical Psychology Review, 31, 736-747. doi:10.1016/j.cpr.2011. 03.001.

Baron, R. M., \& Kenny, D. A. (1986). The moderator-mediator variable distinction in social psychological research: conceptual, strategic, and statistical considerations. Journal of Personality and Social Psychology, 51, 1173-1182. doi:10.1037/0022-3514.51.6.1173.

Benight, C. C., \& Bandura, A. (2004). Social cognitive theory of posttraumatic recovery: the role of perceived self-efficacy. Behaviour Research and Therapy, 42, 1129-1148. doi:10.1016/j.brat.2003. 08.008 .

Brewin, C. R., Dalgleish, T., \& Joseph, S. (1996). A dual representation theory of posttraumatic stress disorder. Psychological Review, 103, 670-686. doi:10.1037/0033-295X.103.4.670.

Bryant, R. A., Salmon, K., Sinclair, E., \& Davidson, P. (2007). A prospective study of appraisals in childhood posttraumatic stress disorder. Behaviour Research and Therapy, 45, 2502-2507. doi:10.1016/ j.brat.2007.04.009.

Canty-Mitchell, J., \& Zimet, G. (2000). Psychometric properties of the multidimensional scale of perceived social support in urban adolescents. American Journal of Community Psychology, 28, 391-400. doi:10.1023/a:1005109522457. 
Cohen, J. A., Deblinger, E., Mannarino, A. P., \& Steer, R. A. (2004). A multisite, randomized controlled trial for children with sexual abuse-related PTSD symptoms. Journal of the American Academy of Child and Adolescent Psychiatry, 43, 393-402. doi:10.1097/ 00004583-200404000-00005.

Dalgleish, T., Meiser-Stedman, R., \& Smith, P. (2005). Cognitive aspects of posttraumatic stress reactions and their treatment in children and adolescents: an empirical review and some recommendations. Behavioural and Cognitive Psychotherapy, 33, 459-486. doi:10. 1017/S1352465805002389.

Ehlers, A., \& Clark, D. M. (2000). A cognitive model of posttraumatic stress disorder. Behaviour Research and Therapy, 38, 319-345. doi: 10.1016/S0005-7967(99)00123-0.

Ehlers, A., Mayou, R. A., \& Bryant, B. (2003). Cognitive predictors of posttraumatic stress disorder in children: results of a prospective longitudinal study. Behaviour Research and Therapy, 41, 1-10. doi:10.1016/S0005-7967(01)00126-7.

Ellis, A. A., Nixon, R. D. V., \& Williamson, P. (2009). The effects of social support and negative appraisals on acute stress symptoms and depression in children and adolescents. British Journal of Clinical Psychology, 48, 347-361. doi:10.1348/014466508x401894.

Foa, E. B., Johnson, K. M., Feeny, N. C., \& Treadwell, K. R. (2001). The child PTSD symptom scale: a preliminary examination of its psychometric properties. Journal of Clinical Child Psychology, 30, 376-384. doi:10.1207/S15374424JCCP3003 9.

Gerard, A. B. (1994). Parent-Child Relationship Inventory (PCRI) Manual. Los Angeles: Western Psychological Services.

Hasan, N., \& Power, T. G. (2004). Children's appraisal of major life events. American Journal of Orthopsychiatry, 74, 26-32. doi:10. 1037/0002-9432.74.1.26.

Hayes, A. F. (2009). Beyond Baron and Kenny: statistical mediation analysis in the new millennium. Communication Monographs, 76, 408-420. doi:10.1080/03637750903310360.

Hayes, A. F. (2013). Introduction to mediation, moderation, and conditional process analysis: A regression-based approach. New York: Guilford Press.

Hayes, A. F., \& Scharkow, M. (2013). The relative trustworthiness of inferential tests of the indirect effect in statistical mediation analysis: does method really matter? Psychological Science, 24, 1918-1927. doi:10.1177/0956797613480187.

Kovacs, M. (1992). The Children's Depression Inventory (CDI). Tonawanda: Multi-Health Systems.

Linley, P. A., \& Joseph, S. (2004). Positive change following trauma and adversity: a review. Journal of Traumatic Stress, 17, 11-21. doi:10. 1023/B:JOTS.0000014671.27856.7e.

Meiser-Stedman, R. (2002). Towards a cognitive-behavioral model of PTSD in children and adolescents. Clinical Child and Family Psychology Review, 5, 217-232. doi:10.1023/a:1020982122107.

Meiser-Stedman, R., Dalgleish, T., Smith, P., Yule, W., \& Glucksman, E. (2007). Diagnostic, demographic, memory quality, and cognitive variables associated with acute stress disorder in children and adolescents. Journal of Abnormal Psychology, 116, 65-79. doi:10. 1037/0021-843x.116.1.65.

Meiser-Stedman, R., Smith, P., Bryant, R., Salmon, K., Yule, W., Dalgleish, T., \& Nixon, R. D. V. (2009). Development and validation of the Child Post-Traumatic Cognitions Inventory (CPTCI). Journal of Child Psychology and Psychiatry, 50, 432440. doi:10.1111/j.1469-7610.2008.01995.x.

Morris, A., Lee, T., \& Delahanty, D. (2013). Interactive relationship between parent and child event appraisals and child PTSD symptoms after an injury. Psychological Trauma: Theory, Research, Practice, and Policy, 5, 554-561. doi:10.1037/a0029894.

Nader, K., Blake, D., Kriegler, J., \& Pynoos, R. (1994). Clinician Administered PTSD Scale for Children (CAPS-C), current and lifetime diagnosis version and instruction manual. Los Angeles: UCLA Neuropsychiatric Institute and National Center for PTSD.

National Institute for Health and Care Excellence. (2005). Post-traumatic Stress Disorder (PTSD): The management of PTSD in adults and children in primary and secondary care [CG26]. London: National Institute for Health and Care Excellence.

Perrin, S., Smith, P., \& Yule, W. (2004). Treatment of PTSD in children and adolescents. In P. M. Barrett \& T. H. Ollendick (Eds.), Handbook of interventions that work with children and adolescents. Prevention and treatment (pp. 217-242). Chichester: Wiley.

Preacher, K., \& Hayes, A. (2004). SPSS and SAS procedures for estimating indirect effects in simple mediation models. Behavior Research Methods, Instruments, \& Computers, 36, 717-731. doi:10.3758/ BF03206553.

Ronan, K., Kendall, P., \& Rowe, M. (1994). Negative affectivity in children: development and validation of a self-statement questionnaire. Cognitive Therapy and Research, 18, 509-528. doi:10.1007/ bf02355666.

Rudy, B. M., Davis, T. E., \& Matthews, R. A. (2012). The relationship among self-efficacy, negative self-referent cognitions, and social anxiety in children: a multiple mediator model. Behavior Therapy, 43, 619-628. doi:10.1016/j.beth.2011.11.003.

Salmon, K., Sinclair, E., \& Bryant, R. A. (2007). The role of maladaptive appraisals in child acute stress reactions. British Journal of Clinical Psychology, 46, 203-210. doi:10.1348/014466506×160704.

Spaccarelli, S. (1994). Stress, appraisal, and coping in child sexual abuse: a theoretical and empirical review. Psychological Bulletin, 116, 340 362. doi:10.1037/0033-2909.116.2.340.

Stallard, P., \& Smith, E. (2007). Appraisals and cognitive coping styles associated with chronic post-traumatic symptoms in child road traffic accident survivors. Journal of Child Psychology and Psychiatry, 48, 194-201. doi:10.1111/j.1469-7610.2006.01692.x.

Trickey, D., Siddaway, A. P., Meiser-Stedman, R., Serpell, L., \& Field, A. P. (2012). A meta-analysis of risk factors for post-traumatic stress disorder in children and adolescents. Clinical Psychology Review, 32, 122-138. doi:10.1016/j.cpr.2011.12.001.

Uhrlass, D. J., Schofield, C. A., Coles, M. E., \& Gibb, B. E. (2009). Selfperceived competence and prospective changes in symptoms of depression and social anxiety. Journal of Behavior Therapy and Experimental Psychiatry, 40, 329-337. doi:10.1016/j.jbtep.2009. 01.001 .

Zimet, G. D., Dahlem, N. W., Zimet, S. G., \& Farley, G. K. (1988). The multidimensional scale of perceived social support. Journal of Personality Assessment, 52, 30-41. doi:10. 1207/s15327752jpa5201 2. 